\title{
Enjeux et promesses de la formation initiale en contexte de médecine ambulatoire et de soins primaires
}

\author{
Stakes and initial teaching promises in the context of ambulatory medicine \\ and primary care
}

Ce numéro de Pédagogie Médicale fait écho de manière très concrète à des initiatives et à des expériences pédagogiques stimulantes qui se développent depuis plusieurs années dans de nombreux milieux académiques francophones de médecine générale, telle qu'on désigne habituellement en Europe la médecine de soins primaires exercée en milieu ambulatoire, ou de médecine de famille, telle qu'on la nomme plus traditionnellement dans d'autres pays, comme par exemple au Québec.

Dans le contexte de nouvelles dispositions réglementaires qui prescrivent désormais à toutes les facultés de médecine françaises d'organiser des stages d'externat en médecine générale au cours du deuxième cycle des études médicales, deux études rapportent une évaluation préliminaire d'expériences pilotes en ce domaine ${ }^{[1,2]}$. Dans un autre contexte, marqué par une longue tradition et par une pratique très expérimentée en la matière, deux contributions québécoises illustrent par ailleurs le souci des enseignants en médecine de famille d'optimiser leurs actions de supervision et d'encadrement en formation post-graduée ${ }^{[3,4]}$.

Ces différentes contributions rappellent fort opportunément que, pour être judicieuse, toute réflexion concernant la problématique de la formation médicale doit nécessairement être conduite en référence à une double préoccupation, de nature politique et pédagogique.

La dimension politique répond à l'idée que l'université médicale doit être consciente qu'elle est dépositaire d'une responsabilité sociale. Boelen et Heck, au nom de l'Organisation mondiale de la santé $^{[5]}$, désignent par là l'exigence que les institutions de formation des professionnels de santé « centrent [leurs] activités d'enseignement, de recherche et de service sur les préoccupations et les besoins prioritaires en matière de santé de la communauté, de la région et de la nation qu'elles ont le mandat de servir, en sachant que ceux-ci devraient être déterminés conjointement avec les gouvernements, les organismes de santé, les professionnels de la santé et le public». Pourtant, dans la plupart des pays industrialisés, la première ligne de soins souffre d'un risque de pénurie en médecins. Une étude française récente confirme à nouveau le désintérêt pour la médecine générale des externes en fin de second cycle ${ }^{[6]}$; cette discipline n'apparaît qu'en avant dernière position de leurs choix et n'est privilégiée positivement que par moins d'un quart des étudiants. Faut-il vraiment s'en étonner alors que la formation médicale pré-graduée reste encore fondamentalement hospitalo-centrée, organisée dans des centres dont le recrutement est de plus en plus spécialisé ? Il est juste de reconnaître que le courant a cependant commencé à s'inverser. 
À cet égard, il est extrêmement stimulant de constater que des tribunes publiées dans les plus prestigieuses revues de recherche clinique ${ }^{[7]}$ n'hésitent plus à militer pour un radical changement de paradigme des programmes de formation initiale, en invitant désormais à recruter les spécialistes dans le cadre d'une sur-spécialisation qui succéderait à une formation initiale conduite en médecine communautaire. Les enjeux sont de taille puisqu'il est désormais bien établi que non seulement l'influence d'un stage d'externat sur le choix de carrière des étudiants était bien réelle, mais aussi que cet impact était encore plus déterminant pour les étudiants attirés par un exercice en soins primaires ${ }^{[8]}$. Nonobstant les limites qu'elles comportent, les expériences rapportées respectivement par Renoux ${ }^{[1]}$, Gelly ${ }^{[2]}$ et leurs collaborateurs sont donc précieuses. Elles confirment à la fois la viabilité, la pertinence et la puissance d'une modélisation précoce, auprès des externes, des rôles professionnels et du mode d'exercice qui devraient concerner au moins la moitié d'entre eux.

Pour pertinent qu'il soit, le contexte de la médecine ambulatoire et des soins primaires ne saurait pour autant suffire à garantir une formation adaptée et efficiente. Encore faut-il qu'il soit aussi pédagogiquement efficacement exploité, de façon judicieuse et avec compétence, pour soutenir les apprentissages des étudiants et les motiver à l'exercice de la discipline. Plusieurs études mettent en garde vis-à-vis du risque qu'un stage effectué au sein de cabinets ayant un fonctionnement non optimal puisse être contreproductif par rapport à l'intention d'attirer les étudiants vers la discipline ${ }^{[9]}$. On doit à Irby d'avoir produit en 1995 la première revue méthodique et systématique dédiée à l'analyse des dispositifs de formation en milieu ambulatoire ${ }^{[10]}$. À l'issue d'une analyse de plus d'une centaine de travaux de recherche dédiés à l'évaluation de tels programmes, il en avait identifié à la fois les forces et les atouts mais aussi les lacunes et les faiblesses. Les déficits concernaient le caractère aléatoire des expériences d'apprentissage, la pauvreté des interactions, leur centration exclusive sur la tâche de soin au détriment de la rétro-action pédagogique, l'absence de conditions favorisant un apprentissage collaboratif. Les forces étaient liées aux caractéristiques des formateurs reconnus comme efficaces, fournissant une supervision effective, à la fois clinique et pédagogique, et agissant comme modèles de rôle explicites. Au terme de son étude, Irby avait formulé plusieurs recommandations : développer les conditions qui optimisent la continuité ou tout au moins le caractère longitudinal des expériences d'apprentissage; former les enseignants cliniciens à la pratique de la supervision pédagogique, de la rétro-action après observation directe et de la modélisation explicite de leurs rôles; encourager la pratique réflexive ainsi que l'apprentissage collaboratif et auto-dirigé ; pour ce faire, créer un environnement favorable en planifiant notamment des moments protégés pour la réflexion et l'apprentissage et aider les étudiants à formuler des objectifs clairs et réalistes concernant les compétences visées, grâce à un tutorat personnalisé développé sur une longue période. Toutes ces orientations demeurent d'une grande validité. Elles ont été consolidées depuis par de multiples travaux de recherche en éducation médicale, qui, au-delà du seul contexte ambulatoire et de soins primaires, leur confèrent authentiquement le statut de standards de qualité pour la formation en contexte clinique. À ce titre, elles auraient aussi avantage à être mieux prises en compte dans les stages d'externat effectués en contexte de médecine hospitalière et spécialisée, comme plusieurs études l'ont bien établi ${ }^{[11]}$. Dans une telle perspective, et même si les usages des dénominations de superviseur, de conseiller pédagogique, de tuteur ou de mentor sont encore, ici ou là, imparfaitement stabilisés, les contributions respectives de Côté, de Giroux et de leurs collaborateurs sont exemplaires de l'aide, irremplaçable et nécessaire, que peuvent apporter les enseignants cliniciens pour soutenir les étudiants dans leurs apprentissages et la construction des compétences attendues d'eux, tant à l'hôpital qu'en milieu ambulatoire.

Pour la médecine générale ou médecine de famille, en tout cas et à n'en pas douter, un tel 
engagement pédagogique d'excellence est bien le corollaire obligé d'une finalité sociale et politique aujourd'hui clairement identifiée.

\section{Philippe Bail}

Département de médecine générale,

Université de Brest (France)

Mailto : philippe-bail@wanadoo.fr

Didier Giet

Département de médecine générale, Université de Liège (Belgique) Mailto :d.giet@ulg.ac.be

\section{Références}

1. Renoux C, Lehr-Drylewicz A-M, Huas D, Lebeau J-P. Évaluation préliminaire d'un stage d'externat expérimental en médecine générale à la faculté de médecine de Tours. Pédagogie Médicale 2009;10:175-183

2. Gelly J, Aubert J-P, Huas D, Eddi A, Baumann L, Legrain $S$, Nougairède M. Acceptabilité et faisabilité de consultations de prévention réalisées par des externes en cabinet de médecine générale. Études CARE-Prev1 \& CARE-Prev2. Pédagogie Médicale 2009;10:185-192

3. Côté L, Bélanger N, Blais J, Desmartis M, Montreuil A. Implantation d'un système de conseillers pédagogiques dans un milieu de formation en médecine familiale : une recherche-action. Pédagogie Médicale 2009;10:161-174

4. Giroux M, Girard G. Favoriser la position d'apprentissage grâce à l'interaction superviseur-supervisé. Pédagogie Médicale 2009;10:193-210

5. Boelen C, Heck J. Définir et mesurer la responsabilité sociale des facultés de médecine. Genève : OMS, 2000.

6. Lefèvre JH, Karila L, Kernéis S, Fiessinger JN, Rouprêt M. Désintérêt des futurs médecins pour la médecine générale. Résultats d'une enquête nationale sur les choix de spécialisation auprès de 1870 externes français. Presse Med 2009, doi:10.1016/j.lpm.2009.09.007.

7. Gibbon W. Medical schools for the health care needs of the 21st century. Lancet 2007;369:2211-3.

8. Ellsbury KE, Carline JD, Irby DM, Stritter FT. Influence of third-year clerkships on medical student specialty preferences. Adv Health Sci Educ 1998;3:177-86.

9. Keirns C, Bosk CL. The unintended consequences of training residents in dysfunctional outpatient settings Acad Med 2008;83:498-502

10. Irby DM. Teaching and learning in ambulatory care settings: a thematic review of the literature. Acad Med 1995;70:898-931.

11. Langevin S, Hivon R. En quoi l'externat ne s'acquitte t-il pas adéquatement de son mandat pédagogique ? Une étude qualitative fondée sur une analyse systématique de la littérature. Pédagogie Médicale 2007;8:7-23. 